\title{
Effect of hydroalcohol extract of lemon (Citrus limon) peel on a rat model of type 2 diabetes
}

\author{
Juan Lv ${ }^{1}$, Lanxiu Cao ${ }^{2 \star}$, Min $\mathrm{Li}^{1}$, Rui Zhang ${ }^{3}, \mathrm{Fu} \mathrm{Bai}^{1}$, Pengfei Wei ${ }^{4}$ \\ ${ }^{1}$ Department of Traditional Chinese Medicine, ${ }^{2}$ Department of Prescription, Basic Medical College, Shanxi University of \\ Traditional Chinese Medicine, Xianyang, $712046,{ }^{3}$ Department of Diabetes, Second Affiliated Hospital of Shanxi University of \\ Traditional Chinese Medicine, ${ }^{4}$ Department of Radiotherapy, First Affiliated Hospital of Shanxi University of Traditional Chinese \\ Medicine, Xianyang, 712000, China
}

*For correspondence: Email: caolanxiuzhjmed@163.com

\begin{abstract}
Purpose: To determine the effect of the hydroalcohol extract of lemon peel (LP) on a rat model of type 2 diabetes (T2D).

Method: The rat model of T2D by injection of streptozotocin was established. The effects of a hydroalcoholic extract of $L P$ was characterised on a rat model of type 2 diabetes based on body weight, food intake, fasting blood glucose (FBG), glucose tolerance test, and insulin tolerance test. Antioxidant activity and oxidative stress were analysed by superoxide dismutase (SOD) and malonaldehyde (MDA) assays.

Results: In acute toxicity studies, administration of LP extract at $2000 \mathrm{mg} / \mathrm{kg}$ orally did not cause any symptoms of poisoning or death after 14 days. The body weight of rats increased after treatment with $L P$ extracts. Food intake in diabetic rats decreased with LP extract treatment. Continual treatment with $L P$ extracts for 35 days significantly reduced blood glucose levels in diabetic rats. Glucose tolerance improved, and insulin resistance was reduced after treatment with LP extracts. SOD and MDA data indicate that treatment with LP extract alleviated the oxidative stress in diabetic rats as well as enhanced the antioxidant activity of liver in a dosage-dependent manner.

Conclusion: $L P$ extract decreased food intake and FBG, but increased body weight in rats. The effect of $L P$ on T2D is likely related to improved antioxidant activity and reduced oxidative stress. Thus, LP extract has potentials for the treatment of T2D.
\end{abstract}

Keywords: Lemon peel, Type 2 diabetes, Antioxidant activity, Glucose tolerance, Insulin tolerance

\begin{abstract}
This is an Open Access article that uses a funding model which does not charge readers or their institutions for access and distributed under the terms of the Creative Commons Attribution License (http://creativecommons.org/licenses/by/4.0) and the Budapest Open Access Initiative (http://www.budapestopenaccessinitiative.org/read), which permit unrestricted use, distribution, and reproduction in any medium, provided the original work is properly credited.
\end{abstract}

Tropical Journal of Pharmaceutical Research is indexed by Science Citation Index (SciSearch), Scopus, International Pharmaceutical Abstract, Chemical Abstracts, Embase, Index Copernicus, EBSCO, African Index Medicus, JournalSeek, Journal Citation Reports/Science Edition, Directory of Open Access Journals (DOAJ), African Journal Online, Bioline International, Open-J-Gate and Pharmacy Abstracts

\section{INTRODUCTION}

Type 2 diabetes (T2D) is a chronic disorder of the metabolism of carbohydrate and lipid, which has long been diagnosed by hyperglycaemia with a high fasting blood glucose (FBG) level. Patients with T2D represent $90 \%$ of all diabetes patients worldwide [1]. Relative to other diseases, T2D has an extremely high occurrence of complications and may even contribute to Alzheimer's disease and depression [2,3]. Insulin resistance and increased oxidative stress are generally accepted as underlying the main pathogenesis of T2D and its complications $[4,5]$. 
T2D is characterised by progressive degeneration of insulin function, accompanied by $\beta$-cell dysfunction that attempts to compensate for insulin resistance [6]. T2D is often associated with high levels of free radicals [7]and attenuated antioxidant function [8]. Interestingly, the oxidant hydrogen peroxide is known to negatively impact glucose transportation capabilities and to stimulate insulin signalling elements [9]. Thus, oxidant stress may cause insulin resistance in mammalian muscle via the p38 MAPK pathway. For hundreds of years, traditional treatments for patients with T2D have utilised medicinal plants that have few side effects. In recent years, medicinal plants have played an important part in new drug discovery. The most widely used antiT2D drug, metformin, was initially developed from Galega officinalis, a medicinal plant that has been used for diabetes treatment for several hundred years [10]. The extract of Galega officinalis provided a predicted chemical structure for new anti-diabetes drugs. Therefore, there has been increasing interest in the research on new medicinal plants that have anti-diabetes properties with few or no side effects to discover chemical structures that account for their therapeutic effect.

Lemon, which is grown worldwide, is used for ethnomedicinal applications due to the antiinflammatory and anti-tumour functions of flavonoids and limonene that are contained in the plant $[11,12]$. Natural antioxidants extracted from plants are attracting more interest for their in vivo radical scavenging activities. The bioactive components that are extracted from lemon peel (LP) depend on the lemon species and the method of extraction.

In this study, the peel of Citrus limon was used for extraction by the hydroalcoholic method. The anti-diabetic and antioxidant effects of LP extract were examined by performing experiments in rat model of T2D.

\section{EXPERIMENTAL}

\section{Plant material and extraction}

Lemon (Citrus limon) was purchased from Shanxi University of traditional Chinese Medicine. The LP was cleaned and dried at 50 ${ }^{\circ} \mathrm{C}$ for $48 \mathrm{~h}$. After grinding into powder, the lemon peel was extracted in $50 \%$ water / $50 \%$ ethanol for $48 \mathrm{~h}$ using a Soxhlet extractor. Water and ethanol in the resultant liquid was removed by lyophilizer. The crude extracts were stored at 4 ${ }^{\circ} \mathrm{C}$.

\section{Experimental animals}

Healthy male Sprague-Dawley (SD) rats with weights ranging from 240 to $280 \mathrm{~g}$ were used in this study. All rats were raised in the same environment with a constant humidity of $50 \%$ at $24^{\circ} \mathrm{C}$ in a 12-h light/dark cycle. All animals were raised and observed in specific pathogen free laboratory animal room. The study was approved by Animal Ethic Committee of the Affiliated Hospital of Shanxi University of Traditional Chinese Medicine (no. EA_20160083), and the experiments with rats were in full compliance with the European Communities Council Directive of 24 November 1986 (86/609/EEC) [13] and with the Guidelines laid down by the NIH in the US [14].

\section{Acute toxicity studies}

Acute toxicity analysis of LP extracts was performed on SD rats according to Organization for Economic Co-operation and Development (OECD) Guidelines for Testing of Chemicals No. 423. Before acute toxicity analysis, SD rats were fasted for $5 \mathrm{~h}$ with only water available, and then divided into four groups with eight animals in each group. The rats were administrated with LP extract at a dosage of $5,50,500$, and 2000 $\mathrm{mg} / \mathrm{kg}$ orally. The rats were observed individually every $24 \mathrm{~h}$ for two weeks. General behaviours, including writhing, gasping, palpitation, decreased respiratory rate, and mortality were observed.

\section{Establishment of rat model ofT2D and animal grouping}

To establish rat model of T2D, rats were adapted to the new environment for 7 days and then supplied with a $10 \%$ fructose solution for 14 days. The rats were then injected with $30 \mathrm{mg} / \mathrm{kg}$ streptozotocin (STZ) in citrate buffer, which causes pancreatic $\beta$-cell dysfunction. Rats in control groups were fed with water followed by injection with citrate buffer only [15]. FBG level was measured with a glucose meter 7 days after T2D induction. Fats with a FBG greater than 250 $\mathrm{mg} / \mathrm{dL}$ were considered to be diabetic.

The SD rats were divided into four groups: normal control (NC), diabetic control (DC), diabetic + low dose of LP extract (DLDLPE, 250 $\mathrm{mg} / \mathrm{kg}$ ), and diabetic + high dose of LP extract (DHDLPE, $500 \mathrm{mg} / \mathrm{kg}$ ). LP extracts of different concentrations were administered orally to rats in the DLDLPE and DHDLPE groups via a forcefeeding needle once a day continuously for 35 days after establishing TD2 models. For NC and DC groups, saline was administered instead. For 
35 days, we recorded body weight, FBG, and food intake of SD rats before and after drug administration.

\section{Glucose tolerance test (GTT)}

To analyse glucose tolerance in SD rats, GTT was performed. After rats were fasted for $12 \mathrm{~h}$, blood glucose was measured. The rats were administered with saline or LP extracts $1 \mathrm{~h}$ prior to glucose administration. After sampling blood from the tail vein, glucose was immediately intraperitoneally injected into rats at a dose of 1 $\mathrm{g} / \mathrm{kg}$. Blood glucose was measured at $0,0.5,1$, 1.5 , and $2 \mathrm{~h}$ after glucose administration.

\section{Insulin tolerance test (ITT)}

After the GTT was performed on overnight-fasted rats daily for 4 days. The rats were administered saline or LP extracts $1 \mathrm{~h}$ before insulin administration. After sampling blood from the tail vein, a single dose of insulin solution $(0.5 \mathrm{U} / \mathrm{kg})$ was immediately subcutaneously injected into each rat. Blood glucose was tested at $0,0.5,1$, and $1.5 \mathrm{~h}$ after insulin injection.

\section{Histological assessment}

After $24 \mathrm{~h}$ fixation, pancreas tissues were dehydrated, cleared in xylene, and immersed in paraffin for embedding. The embedded tissue was sliced by microtome, dehydrated, and dewaxed. The slices were then stained with haematoxylin, washed with distilled water, decolorized using hydrochloric acid ethanol, and then stained again with eosin. Slices were then dehydrated, dried, and sealed for observation under microscope.

\section{Assessment of oxidative stress and antioxidant activity}

Superoxide dismutase (SOD) and malonaldehyde (MDA) extractions were performed by phosphate-buffered saline after the liver tissues was ground by liquid nitrogen grinding. SOD activity was analysed by a commercial kit (Beyotime, China) and expressed as units/mg protein. MDA content was analysed using a commercialMDA assay kit (Beyotime, China) and expressed as $\mu \mathrm{mol} / \mathrm{mg}$ protein. The concentration of total protein was measured by Bradford protein kit (Beyotime, China) [16].

\section{Statistical analysis}

The data was analysed using SPSS 21 software (IBM, USA). The results are presented as mean \pm standard deviation. Comparisons between two groups were performed by Student's $t$-test. $P<$ 0.05 indicated statistical significance.

\section{RESULTS}

\section{Acute toxicity}

In acute toxicity analysis, after 14 days of orally administering LP extract at $2000 \mathrm{mg} / \mathrm{kg}$, any symptoms of poisoning or death were not observed, indicating that LP extract was not toxic for rats at $2000 \mathrm{mg} / \mathrm{kg}$. Therefore, oral dosages of 250 and $500 \mathrm{mg} / \mathrm{kg}$ were safe and feasible for this study.

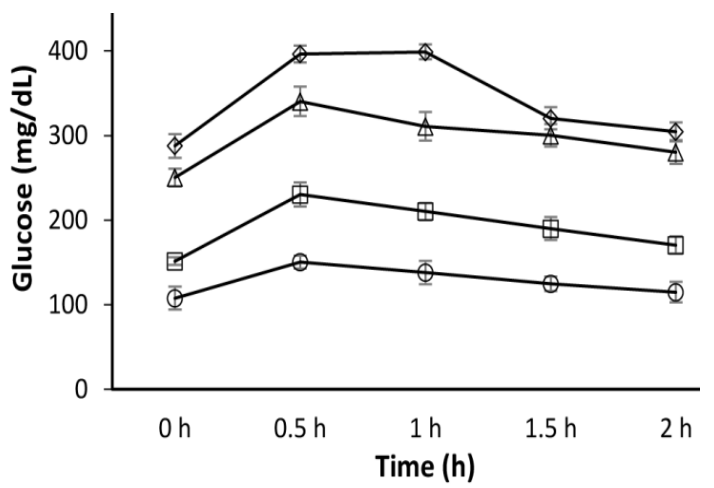

Figure 1: Effect of LP extracts on glucose tolerance. $O$ represents NC group; $\diamond=D C$ group (T2D rats treated with vehicle); $\Delta=$ DLDLPE group (T2D rats treated with a low dose of LP extracts); $\square$ represents DHDLPE group (T2D rats treated with a high dose of LP extracts)

\section{Body weight, FBG, and food intake}

Body weight, FBG, and food intake was evaluated for 35 days, before and after administration of LP extract or saline. Body weight was found to decrease after establishing the rat model of T2D (Table 1). During saline administration, the body weight of diabetic rats continued to decrease. However, after treatment with LP extracts, the body weight of rats increased in the DLDLPE and DHDLPE groups. A high dose of LP extract $(500 \mathrm{mg} / \mathrm{kg})$ increased body weight more effectively than a low dose of LP extract $(250 \mathrm{mg} / \mathrm{kg})$. Food intake also increased after establishing a model of T2D in rats. After treatment with LP extracts, food intake decreased in diabetic rats. However, a high dose of LP extract did not reduce food intake more than a low dose of LP extract. The rats in the DC group had a significantly higher FBG level $(p<$ 0.01 ) than the NC group. Continuation of treatment with LP extracts for 35 days significantly reduced blood glucose levels of diabetic rats. 
Table 1: Effect of LP extract treatment on food intake and body weight before and after drug administration in STZ-induced diabetic rats. NC, Normal rats treated with vehicle alone; DC, T2D rats treated with vehicle alone; DLDLPE, T2D rats treated with a low dose of LP extracts $(250 \mathrm{mg} / \mathrm{kg})$; DHDLPE, T2D rats treated with a high dose of LP extracts $(500 \mathrm{mg} / \mathrm{kg})$

\begin{tabular}{llllll}
\hline Parameter & & NC & DC & DLDLPE & DHDLPE \\
\hline Body weight $(\mathrm{g})$ & Before & $269.0 \pm 5.2$ & $220.0 \pm 7.7$ & $224.0 \pm 6.5$ & $225.0 \pm 8.7$ \\
& After & $288.0 \pm 4.5$ & $170.0 \pm 5.2$ & $250.0 \pm 3.8$ & $265.0 \pm 5.3$ \\
Food intake & Before & $17.5 \pm 2.1$ & $30.5 \pm 2.5$ & $29.2 \pm 1.2$ & $31.5 \pm 2.1$ \\
(g/rat/day) & After & $19.2 \pm 1.8$ & $33.4 \pm 2.2$ & $22.4 \pm 1.7$ & $26.3 \pm 2.6$ \\
FBG & Before & $100.2 \pm 5.7$ & $283.8 \pm 7.5$ & $280.6 \pm 15.3$ & $277.7 \pm 8.6$ \\
(mg/dl) & After & $109.8 \pm 10.5$ & $288.4 \pm 12.8$ & $180.6 \pm 6.5$ & $155.1 \pm 12.2$ \\
\hline
\end{tabular}

\section{GTT and ITT}

In the GTT, the blood glucose level of rats in the NC, DLDLPE, and DHDLPE groups reached a maximum value at $0.5 \mathrm{~h}$ and then decreased over time. However, rats in the DC group reached a maximum blood glucose value at $1 \mathrm{~h}$. In the ITT, after insulin administration, FBG initially decreased and then increased in the DLDLPE and DHDLPE groups, when compared to the DC group. After insulin administration for 2 $\mathrm{h}$, the FBG level in the DLDLPE group was close to that of the DC group. In the NC and DHDLPE groups, FBG reached a minimum value $0.5 \mathrm{~h}$ after insulin administration. In the DC and DLDLPE groups, FBG level reached a minimum value $1 \mathrm{~h}$ after insulin administration.

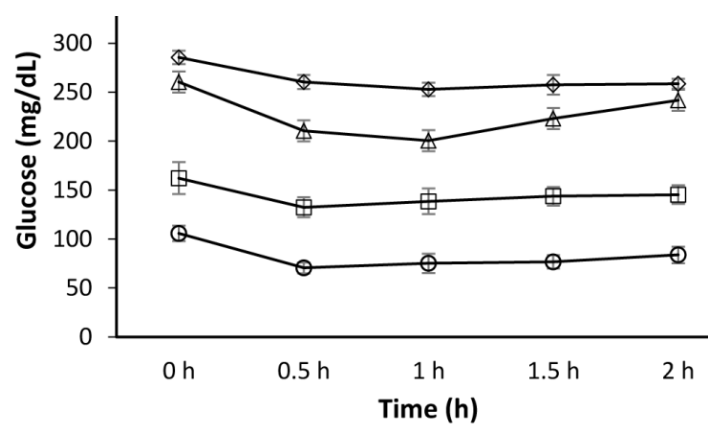

Figure 2: Effect of LP extracts on insulin tolerance. $\bigcirc$ = NC group; $\diamond$ represents DC group (T2D rats treated with vehicle); $\Delta$ represents DLDLPE group (T2D rats treated with a low dose of LP extracts); $\square=$ DHDLPE group (T2D rats treated with a high dose of LP extracts)

\section{Histological features}

Haematoxylin-eosin staining results showed serious damage to the intra-pancreatic islets and the number and size of islets was decreased in STZ-induced diabetic rats (Figure $3 \mathrm{~A}$ and Figure 3B). The vacuolation and invasion of intrapancreatic tissue in the diabetic rats were also observed. After treatment with LP extract, the regeneration of intrapancreatic islet cells and enhanced restoration of intrapancreatic tissues were observed (Figure $3 \mathrm{C}$ and Figure $3 \mathrm{D}$ ).

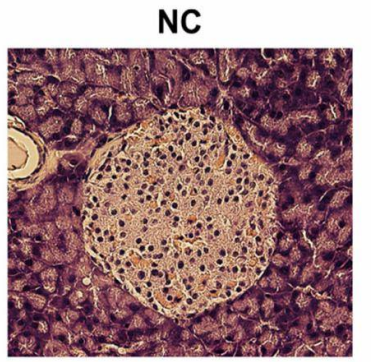

DLDLPE
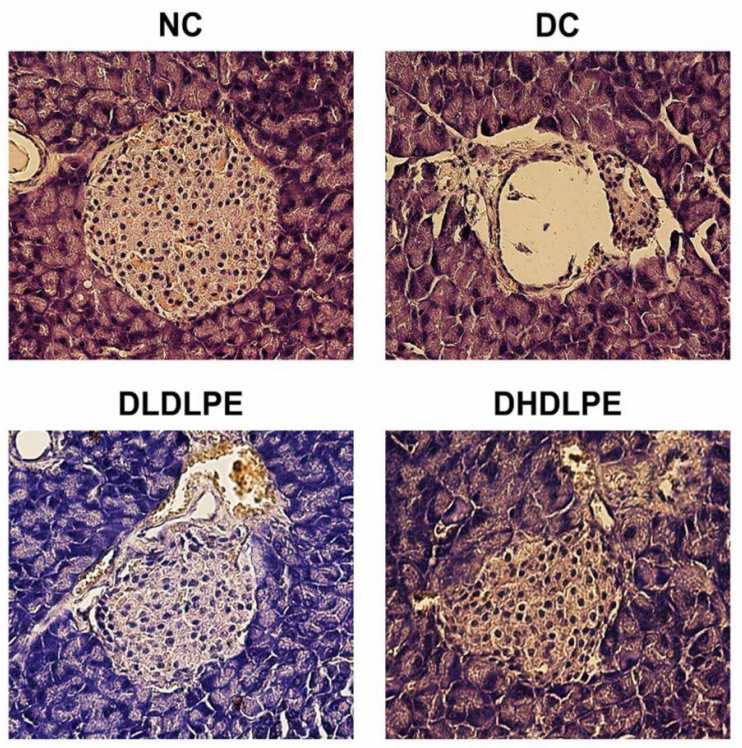

DHDLPE

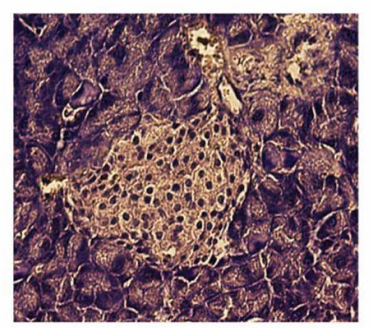

Figure 3: Effect of treatment with LP extracts on pancreatic islet tissues of rats in T2D model and control groups. NC, Normal rats treated with vehicle alone; DC, T2D rats treated with vehicle alone; DLDLPE, T2D rats treated with a low dose of LP extracts $(250 \mathrm{mg} / \mathrm{kg})$; DHDLPE, T2D rats treated with a high dose of LP extracts $(500 \mathrm{mg} / \mathrm{kg}$ )

\section{Antioxidant activity and oxidative stress}

The antioxidant activity and oxidative stress were determined by measuring MDA level and SOD activity, respectively. The increase of MDA was observed in diabetic rats, indicating that the oxidative stress in rat liver significantly increased after T2D model establishment $(p<0.05)$ (Figure $4 \mathrm{~A})$. The oxidative stress, as measured by SOD activity, was alleviated in diabetic rats after treatment with a high dose of LP extract. However, low doses of LP extract did not have the same effect on oxidative stress as high doses. The decreased SOD activity in diabetic rats indicated that antioxidant activity decreased in the liver after T2D model establishment, and that treatment with LP extract restored antioxidant activity in a dose-dependent manner $(p<0.05)$ (Figure 4B). 


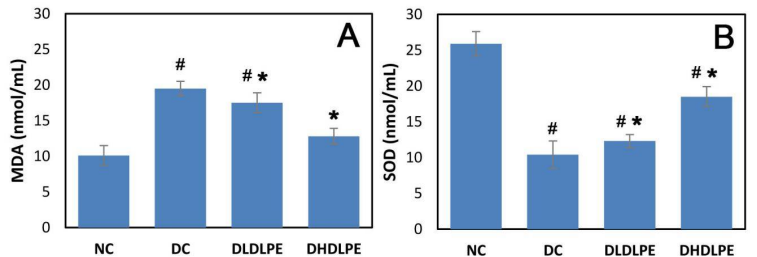

Figure 4: Effect of LP extracts on MDA (A) and SOD (B) of liver tissues; ${ }^{\#} p<0.05$ compared with NC group; ${ }^{*} p<0.05$ compared with DC group. NC, Normal rats treated with vehicle alone; DC, T2D rats treated with vehicle alone; DLDLPE, T2D rats treated with a low dose of LP extracts $(250 \mathrm{mg} / \mathrm{kg})$; DHDLPE, T2D rats treated with a high dose of LP extracts $(500 \mathrm{mg} / \mathrm{kg})$

\section{DISCUSSION}

Lemon has been used as a traditional medicine, likely due to the anti-inflammatory and antitumour functions of flavonoids and limonene that are contained in the plant $[11,12]$. In this study, the anti-diabetic and antioxidant activities of extracts of lemon peel was evaluated. Type 2 diabetes is a chronic disorder of energy metabolism, which is diagnosed by hyperglycaemia with a consistently high FBG level. In this study, STZ-induced T2D rat model was used to assess the effect of treatment with LP extract on T2D.The acute toxicity of LP extract and the effect of LP extract on FBG, body weight, food intake, GTT, ITT, histological changes, and oxidative stress were tested.

Reduced body weight is a representative symptom for T2D in rats [17]. The establishment of a T2D rat model caused reduced body weight in this study. Reduction of body weight was rescued in DLDLPE and DHDLPE groups, indicating that treatment with LP extracts increased the body weight of diabetic rats. Treatment with LP extracts also reduced food intake and FBG in diabetic rats. These results suggest that LP extracts may alleviate typical T2D symptoms. In previous studies, the antidiabetes mechanisms of natural plant medicines were found to be involved in the maintenance of glucose homeostasis, gastrointestinal glucose absorption, insulinotropic actions, and promoting pancreatic $\beta$-cell regeneration [18-20].

Furthermore, the results of GTT and ITT showed that treatment with LP extracts improved glucose tolerance and reduced insulin resistance. Histological analysis showed that LP extracts restored the structure of and helped regenerate intra-pancreatic islets. SOD and MDA analyses indicated that treatment with LP extract alleviated oxidative stress in diabetic rats' and restored antioxidant activities in the liver in a dosedependent manner. It was reported that persistent hyperglycaemia destroys antioxidant balance by reducing antioxidant levels and producing reactive oxygen species [21,22], which is consistent with the result of this study (Figure 4). The results suggest that treatment with LP extract may alleviate T2D symptoms by reducing oxidative stress and restoring antioxidant activity.

\section{CONCLUSION}

The findings of this study show that treatment of type 2 diabetic rats with LP extract ameliorates T2D by reducing food intake and FBG, and increasing body weight. Glucose tolerance and insulin tolerance are also improved after LP administration. The effect of treatment with LP extract is related to increased antioxidant activity and reduced oxidative stress.

\section{DECLARATIONS}

\section{Conflict of Interest}

No conflict of interest associated with this work.

\section{Contribution of Authors}

We declare that this work was done by the authors named in this article and all liabilities pertaining to claims relating to the content of this article will be borne by the authors. Lanxiu Cao designed all the experiments and revised the paper. Juan Lv, Min Li, Rui Zhang and Fu Bai performed the experiments, and Pengfei Wei and Juan Lv wrote the paper.

\section{REFERENCES}

1. Mohammed A, Md S. Butanol fraction of Khaya senegalensis root modulates $\beta$-cell function and ameliorates diabetes-related biochemical parameters in a type 2 diabetes rat model. J Ethno pharmacol 2014;154(3): 832-838.

2. Pouwer F, Beekman AT, Nijpels G, Dekker J, Snoek FJ, Kostense $P$, Heine $R$, Deeg $D$. Rates and risks for comorbid depression in patients with Type 2 diabetes mellitus: results from a community-based study. Diabetologia 2003; 46(7): 892-898.

3. Ahtiluoto $S$, Polvikoski $T$, Peltonen $M$, Solomon $A$, Tuomilehto J, Winblad $B$, Sulkava $R$, Kivipelto $M$. Diabetes, Alzheimer disease, and vascular dementia $A$ population-based neuropathologicstudy. Neurology 2010; 75(13): 1195-1202.

4. Pistrosch F, Passauer J, Fischer S, Fuecker K, Hanefeld $M$, Gross $P$. In type 2 diabetes, rosiglitazone therapy for insulin resistance ameliorates endothelial dysfunction independent of glucose control. Diabetes Care 2004; 27(2): 484-490. 
5. Maritim AC, Sanders RA. Diabetes, oxidative stress, and antioxidants: a review. J Biochem Mol Toxicol 2003; 17(1): 24-38.

6. Stumvoll M, Goldstein BJ, Haeften TWV: Pathogenesis of type 2 diabetes mellitus. Med Clin N Am 2004; 88(4): 787-835.

7. Baynes JW. Role of oxidative stress in development of complications in diabetes. Diabetes 1991; 40(4): 405412.

8. Saxena AK, Srivastava P, Kale RK, Baquer NZ. Impaired antioxidant status in diabetic rat liver. Effect of vanadate. Biochem Pharmacol 1993; 45(3): 539-542.

9. Archuleta $T L$, Lemieux AM, Saengsirisuwan V, Teachey MK, Lindborg KA, Kim JS, Henriksen EJ. Oxidant stress-induced loss of IRS-1 and IRS-2 proteins in rat skeletal muscle: role of p38 MAPK. Free Radical Biol Med 2009; 47(10): 1486-1493.

10. Cragg GM, Newman DJ. Natural products: a continuing source of novel drug leads. Biochim Biophys Acta 2013; 1830(6): 3670-3695.

11. Jr ME, Kandaswami C, Theoharides TC. The effects of plant flavonoids on mammalian cells: implications for inflammation, heart disease, and cancer. Pharmacol Rev 2000; 52(4): 673-751.

12. Huang YS, Suchen $H$. Polymethoxy flavones are responsible for the anti-inflammatory activity of citrus fruit peel. Food Chem 2010; 119(3): 868-873.

13. European Union Commission Regulation. EC 86/609/EEC. Off. J. Eur. Union 1986, L358, 1-28.

14. National Institutes of Health. Guidelines regarding the care and use of animals for experimental procedures (LIN-DL-11) NIH Publication No.8 5-23, revised 1985
15. Wilson RD, Islam MS: Fructose-fed streptozotocininjected rat. an alternative model for type 2 diabetes. Pharmacol Rep 2012; 64(1): 129-139.

16. Bradford MM. A rapid and sensitive method for the quantitation of microgram quantities of protein utilizing the principle of protein-dye binding. Anal Biochem1976; 72(1-2): 248-254.

17. Haidari F, Shahi MM, Zarei M, Rafiei H, Omidian K. Effect of green tea extract on body weight, serum glucose and lipid profile in streptozotocin-induced diabetic rats. A dose response study. Saudi Med J 2012; 33(2): 128133.

18. Gin H, Rigalleau V. Post-prandial hyperglycemia. postprandial hyperglycemia and diabetes. Diabetes Metab2000; 26(4): 265-272.

19. Musabayane CT, Bwititi PT, Ojewole JA. Effects of oral administration of some herbal extracts on food consumption and blood glucose levels in normal and streptozotocin-treated diabetic rats. Methods Find Exp ClinPharmacol2006; 28(4): 223-228.

20. Islam MS, Choi H. Dietary red chilli (Capsicum frutescens L.) is insulinotropic rather than hypoglycemic in type 2 diabetes model of rats. Diabetes Res ClinPract2008; 22(8): 1025-1029.

21. Aragno M, Mastrocola R, Catalano MG, Brignardello E, Danni O, Boccuzzi G. Oxidative stress impairs skeletal muscle repair in diabetic rats. Diabetes2004; 53(4): 1082-1088.

22. Nishikawa $T$, Edelstein $D, D u \quad X L$, Yamagishi $S$, Matsumura T, Kaneda $Y$, Yorek MA, Beebe D, Oates $P J$, Hammes HP. Normalizing mitochondrial superoxide production blocks three pathways of hyperglycaemic damage. Nature 2000; 404(6779): 787-790. 